\title{
Association between serum uric acid and bone health in general population: a large and multicentre study
}

\author{
Xianfeng Lin ${ }^{1, *}$, Chenchen Zhao ${ }^{1, *}$, An Qin ${ }^{2, *}$, Dun Hong ${ }^{3}$, Wenyue Liư ${ }^{4}$ Kangmao \\ Huang ${ }^{1}$, Jian $\mathrm{Mo}^{1}$, Hejun $\mathrm{Yu}^{1}$, Shengjie $\mathrm{Wu}^{5}$ and Shunwu Fan ${ }^{1}$ \\ ${ }^{1}$ Department of Orthopedics Surgery, Sir Run Run Shaw Hospital, Medical College of Zhejiang University, Hangzhou, China \\ ${ }^{2}$ Department of Orthopedics, Shanghai Key Laboratory of Orthopedic Implant, Shanghai Ninth People's Hospital, Shanghai \\ Jiaotong University School of Medicine, Shanghai, China \\ ${ }^{3}$ Department of Orthopedics Surgery, Taizhou Hospital of Wenzhou Medical University, Linhai, China \\ ${ }^{4}$ Department of Endocrinology, The First Affiliated Hospital of Wenzhou Medical University, Wenzhou, China \\ ${ }^{5}$ Department of Cardiology, The Key Laboratory of Cardiovascular Disease of Wenzhou The First Affiliated Hospital of \\ Wenzhou Medical University, Wenzhou, China \\ * These authors have contributed equally to this work \\ Correspondence to: Shunwu Fan, email: shunwu_fan@126.com \\ Shengjie Wu, email: wusj120@163.com \\ Keywords: serum uric acid, bone health, bone mineral density, bone mass loss, DXA, Gerotarget \\ Received: June 30,2015 Accepted: October 06, $2015 \quad$ Published: October 19, 2015
}

This is an open-access article distributed under the terms of the Creative Commons Attribution License, which permits unrestricted use, distribution, and reproduction in any medium, provided the original author and source are credited.

\section{ABSTRACT}

Previous studies proposed that serum uric acid (UA), an endogenous antioxidant, could be a protective factor against bone loss. However, recently, a study with a population of US adults did not note the protective effects of serum UA. Therefore, the exact association between serum UA and bone health remains unclear. We performed a retrospective consecutive cohort study in a Chinese population to examine the association between serum UA and bone health. This cross-sectional study included 17,735 individuals who underwent lumbar spine bone mineral density (BMD) measurements as part of a health examination. In covariance analyses (multivariableadjusted), a high serum UA level was associated with a high BMD, T-score, and Z-score. In binary logistic regression analyses (multivariable-adjusted), a high serum UA level was associated with low odds ratios (ORs) for at least osteopenia and osteoporosis in male (age $\geq 50$ years) $(O R=0.72-0.60$ and $O R=0.49-0.39$, respectively) and postmenopausal female participants $(O R=0.61-0.51$ and $O R=$ 0.66-0.49, respectively). In conclusion, serum UA is associated with BMD, the T-score, and the Z-score, and has a strong protective effect against at least osteopenia and osteoporosis.

\section{INTRODUCTION}

Uric acid (UA) is the final breakdown product of purine metabolism in humans, and it has been traditionally considered to be a risk factor for hypertension, diabetes mellitus, stroke, cardiovascular disease, renal disease, and metabolic syndrome [1-5]. High amounts of serum UA could lead to the deposition of urate crystals, causing gouty arthritis. However, studies have shown that serum UA might function as an antioxidant against oxidative stress in nervous system diseases such as multiple sclerosis, amyotrophic lateral sclerosis, Alzheimer disease, and dementia [6-9].

Osteoporosis and osteopenia are some of the most common diseases worldwide, and they increase the risk of fractures and are major causes of bone fragility [10, 11]. Many studies have suggested that oxidative stress and low circulating levels of antioxidants are associated with reduced bone mineral density (BMD) and osteoporosis [12-15]. In recent years, studies have assessed whether serum UA is a protective factor against bone loss [1622]. Since Nabipour et al. first reported that high serum 
UA levels were associated with high BMD values at all skeletal sites and with low a prevalence of fractures in elderly Australian men (age $\geq 70$ years), many studies have shown that high serum UA levels have beneficial effects on bone metabolism in Korean and Japanese perimenopausal and postmenopausal women and in Korean men (age $\geq 50$ years) [17-21]. Lane et al. proposed that high serum UA levels were associated with a reduction in the risk of incident non-spine fractures in US men (age $\geq 65$ years) [22]. However, the study by Zhang D et al. did not find that high serum UA levels have a protective effect on bone health in US adults (age $\geq 30$ years) [16]. Furthermore, their results were confirmed in a rodent model of chronic mild hyperuricemia, demonstrating that the serum UA level was not associated with BMD and bone biomechanical properties.

Therefore, the exact association between serum UA and bone health remains unclear, and a study involving a large multicenter population is required to clarify the association. The present study aimed to investigate the relationship between serum UA and bone loss, including $\mathrm{BMD}$, the T-score, the Z-score, osteoporosis, and osteopenia, in a large multicenter Chinese population.

\section{RESULTS}

\section{Baseline characteristics}

A total of 22,409 participants were enrolled. According to the exclusion criteria, 2,503 male and 2,171 female participants were eliminated. A total of 17,735 participants (10,596 males and 7139 females) with available serum UA and dual-energy X-ray absorptiometry (DXA) measurement data were finally included in this study (Figure 1). The baseline characteristics of the study population are presented in Table 1 . The mean serum UA

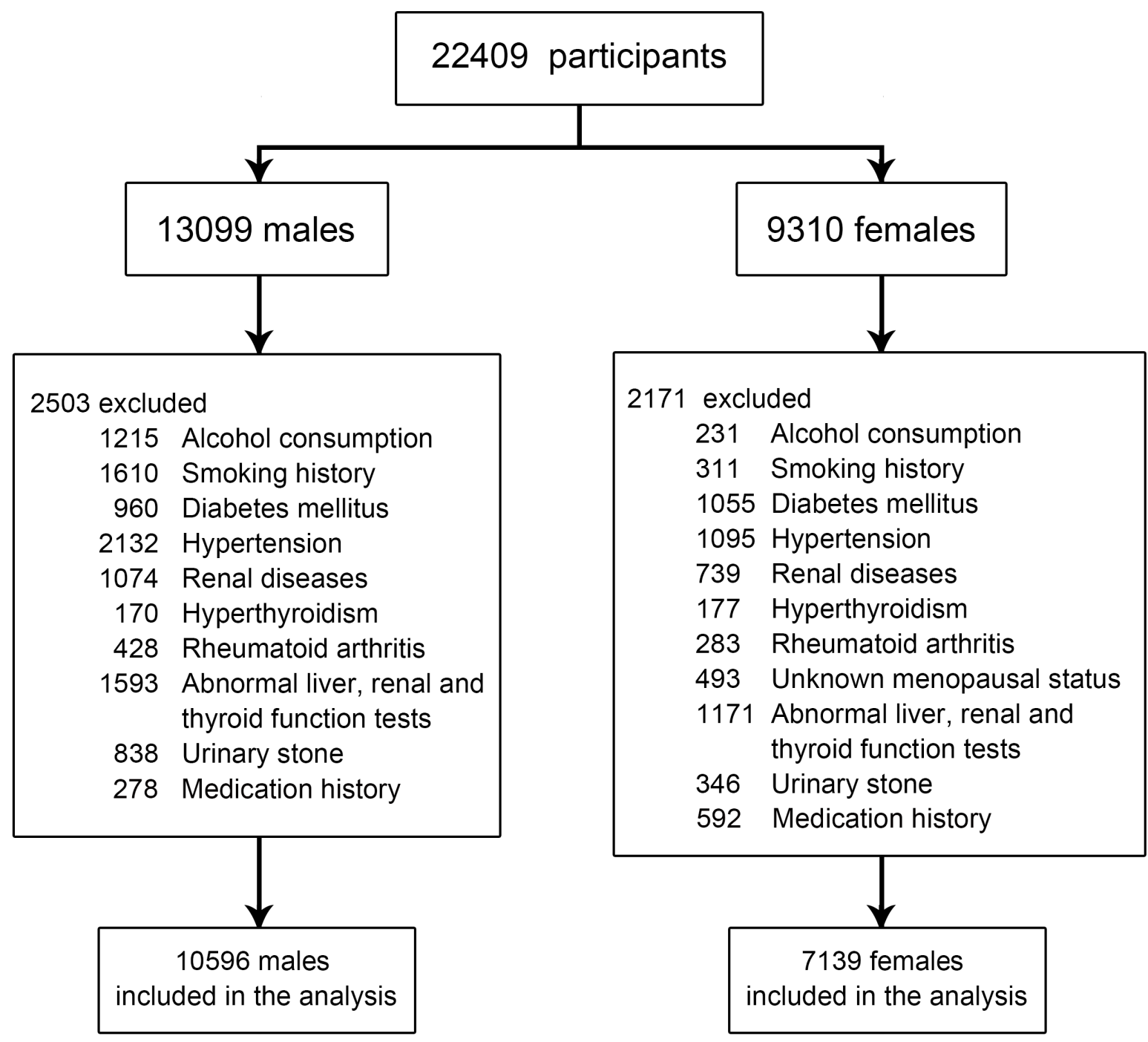

Figure 1: Flow chart of study inclusion. A total of 22,409 participants were enrolled. According to the exclusion criteria, 4,674 participants were excluded. Finally, 17,735 participants (10,596 male and 7,139 female participants) were included. 
Table 1: Clinical and biochemical characteristics of the study cohort grouped as males and females

\begin{tabular}{|c|c|c|c|}
\hline & $\begin{array}{l}\text { All participants } \\
(n=17735)\end{array}$ & \begin{tabular}{|l} 
Males \\
$(n=10596)$
\end{tabular} & \begin{tabular}{|l|} 
Females \\
$(n=7139)$
\end{tabular} \\
\hline $\mathrm{UA}(\mathrm{mmol} / \mathrm{L})$ & $330.57 \pm 94.03$ & $374.80 \pm 82.76$ & $263.79 \pm 66.62$ \\
\hline $\mathrm{BMD}(\mathrm{g} / \mathrm{cm} 2)$ & $1.12 \pm 0.17$ & $1.14 \pm 0.15$ & $1.10 \pm 0.18$ \\
\hline T-score & $-0.62 \pm 1.42$ & $-0.47 \pm 1.36$ & $-0.84 \pm 1.50$ \\
\hline Z-score & $0.14 \pm 1.34$ & $0.04 \pm 1.44$ & $0.29 \pm 1.17$ \\
\hline Age (years) & $49.62 \pm 10.46$ & $49.38 \pm 11.13$ & $49.98 \pm 10.80$ \\
\hline Height (cm) & $164.06 \pm 8.20$ & $168.61 \pm 6.29$ & $157.31 \pm 5.65$ \\
\hline Weight (kg) & $65.48 \pm 11.30$ & $70.63 \pm 10.09$ & $57.84 \pm 8.28$ \\
\hline BMI (kg/m2) & $24.23 \pm 3.14$ & $24.81 \pm 2.99$ & $23.38 \pm 3.16$ \\
\hline Systolic BP (mmHg) & $123.42 \pm 16.31$ & $125.00 \pm 15.39$ & $120.81 \pm 12.29$ \\
\hline Glucose $(\mathrm{mmol} / \mathrm{L})$ & $5.56 \pm 1.41$ & $5.65 \pm 1.15$ & $5.44 \pm 1.22$ \\
\hline Serum calcium $(\mathrm{mmol} / \mathrm{L})$ & $2.36 \pm 0.13$ & $2.37 \pm 0.13$ & $2.35 \pm 0.13$ \\
\hline Serum phosphate $(\mathrm{mmol} / \mathrm{L})$ & $1.11 \pm 0.17$ & $1.08 \pm 0.17$ & $1.17 \pm 0.16$ \\
\hline Alkaline phosphatase (U/L) & $74.25 \pm 21.85$ & $75.98 \pm 19.95$ & $71.63 \pm 24.21$ \\
\hline $\operatorname{AST}(\mathrm{U} / \mathrm{L})$ & $25.30 \pm 18.65$ & $26.79 \pm 21.19$ & $23.06 \pm 13.65$ \\
\hline $\operatorname{ALT}(\mathrm{U} / \mathrm{L})$ & $29.08 \pm 36.84$ & $34.13 \pm 42.59$ & $21.44 \pm 23.88$ \\
\hline Triglycerides (mmol/L) & $1.78 \pm 1.48$ & $2.04 \pm 1.64$ & $1.39 \pm 1.07$ \\
\hline Total cholesterol $(\mathrm{mmol} / \mathrm{L})$ & $5.08 \pm 0.98$ & $5.09 \pm 0.97$ & $5.07 \pm 0.99$ \\
\hline Serum urea nitrogen $(\mathrm{mmol} / \mathrm{L})$ & $5.16 \pm 1.34$ & $5.35 \pm 1.31$ & $4.88 \pm 1.33$ \\
\hline Serum creatinine $(\mu \mathrm{mol} / \mathrm{L})$ & $77.85 \pm 18.95$ & $85.14 \pm 15.22$ & $66.85 \pm 18.73$ \\
\hline Total bilirubin $(\mu \mathrm{mol} / \mathrm{L})$ & $13.67 \pm 5.87$ & $14.61 \pm 6.25$ & $12.24 \pm 4.92$ \\
\hline Total protein $(\mathrm{g} / \mathrm{L})$ & $76.20 \pm 4.39$ & $75.96 \pm 4.34$ & $76.58 \pm 4.44$ \\
\hline eGFR (mL/min/1.73m2) & $90.41 \pm 17.93$ & $90.60 \pm 16.13$ & $90.12 \pm 20.34$ \\
\hline
\end{tabular}

UA, uric acid; BP, blood pressure; AST, aspartate transaminase; ALT, alanine aminotransferase; BMD, bone mineral density; eGFR, estimated glomerular filtration rate.

levels in males and females were $374.80 \pm 82.76$ and $263.79 \pm 66.62 \mathrm{mmol} / \mathrm{L}$, respectively. The mean BMD and T-score were higher in male participants than in female participants $(1.14 \pm 0.15$ vs. $1.10 \pm 0.18, P=0.000$ and $-0.47 \pm 1.36$ vs. $-0.84 \pm 1.50, P=0.000$, respectively).

\section{Relationship between serum UA levels and BMD}

The participants were divided into four quartiles (Q1, Q2, Q3, and Q4) according to the serum UA levels. Before adjusting for potential confounders, BMD was found to significantly increase across the quartiles in males and postmenopausal females, and a non-significant inverse trend was observed in females (Figure 2a). A covariance analysis was performed to investigate the association between serum UA levels and BMD after controlling for confounders. After adjusting for age; height; weight; systolic blood pressure (BP); levels of serum glucose, total protein, blood urea nitrogen, serum calcium, serum phosphorus, alkaline phosphatase, alanine aminotransferase (ALT), aspartate aminotransferase (AST), triglycerides, total cholesterol, total bilirubin, and serum creatinine; and the estimated glomerular filtration rate (eGFR), BMD was found to have an increasing trend across the quartiles in male, female, and postmenopausal female participants (Figure 2b). BMD and serum UA levels were affected by obesity; therefore, the participants were divided into subgroups according to body mass index (BMI; $\mathrm{BMI}<25$ and $\mathrm{BMI} \geq 25$ ), and the increasing trend was confirmed in the subgroups (Figure $2 \mathrm{c}$ and $2 \mathrm{~d}$ ).

\section{Relationship of serum UA levels with the T-score and $\mathrm{Z}$-score}

Before adjusting for potential confounders, the T-score was found to significantly increase across the quartiles in males (age $\geq 50$ years) and postmenopausal females (Figure 3a). After adjusting for potential confounders, the T-score was found to increase across the quartiles in males and postmenopausal females, although the change in postmenopausal females was not significant $(P=0.053)$ (Figure 3b). Additionally, before adjusting for potential confounders, the Z-score was found to have no remarkable increasing trend across the quartiles in male (age $<50$ years) and premenopausal female participants (Figure 3c). However, after adjusting for potential confounders, the Z-score was found to significantly increase across the quartiles in males (age $<50$ years) $(P$ $=0.049$; Figure 3d). 


\section{Prevalence of at least osteopenia and osteoporosis in the serum UA quartiles}

Univariate analysis showed that the odds ratios (ORs) for both at least osteopenia and osteoporosis decreased significantly from Q1 to Q4 (Supplemental Tables 1 and 2). Additionally, after adjusting for potential confounders, the multivariate analysis revealed that the ORs for both at least osteopenia and osteoporosis decreased significantly from Q1 to Q4 in males (age $\geq$ 50 years) in a dose-dependent manner (Figure $4 \mathrm{a}$ and Supplemental Table 3). Among the male participants, compared to the OR for at least osteopenia in Q1, the ORs were 40\% lower in Q4, 33\% lower in Q3, and 28\% lower in Q2. The ORs and 95\% confidence intervals (CI) for osteoporosis among the male participants in Q2, Q3, and Q4 were 0.395 (0.245-0.637), 0.491 (0.295-0.818), and $0.386(0.220-0.678)$, respectively (Figure $4 \mathrm{~b}$ and Supplemental Table 3). Additionally, the ORs and 95\% $\mathrm{CI}$ for at least osteopenia and osteoporosis among the postmenopausal female participants in Q4 were 0.515 (0.299-0.889) and 0.494 (0.299-0.818), respectively (Figures $4 \mathrm{a}$ and $4 \mathrm{~b}$, Supplemental Table 4). Moreover, the ORs and $95 \%$ CI for osteoporosis among the postmenopausal female participants in Q2 and Q3 were $0.662(0.366-1.058)$ and $0.551(0.328-0.928)$, respectively (Figure 4b, Supplemental Table 4), which are consistent with the above results. A stratified analysis for obesity $(\mathrm{BMI}<25$ and $\mathrm{BMI} \geq 25)$ showed a successive decrease in ORs in males (age $\geq 50$ years) and postmenopausal

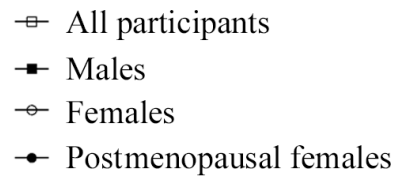

a
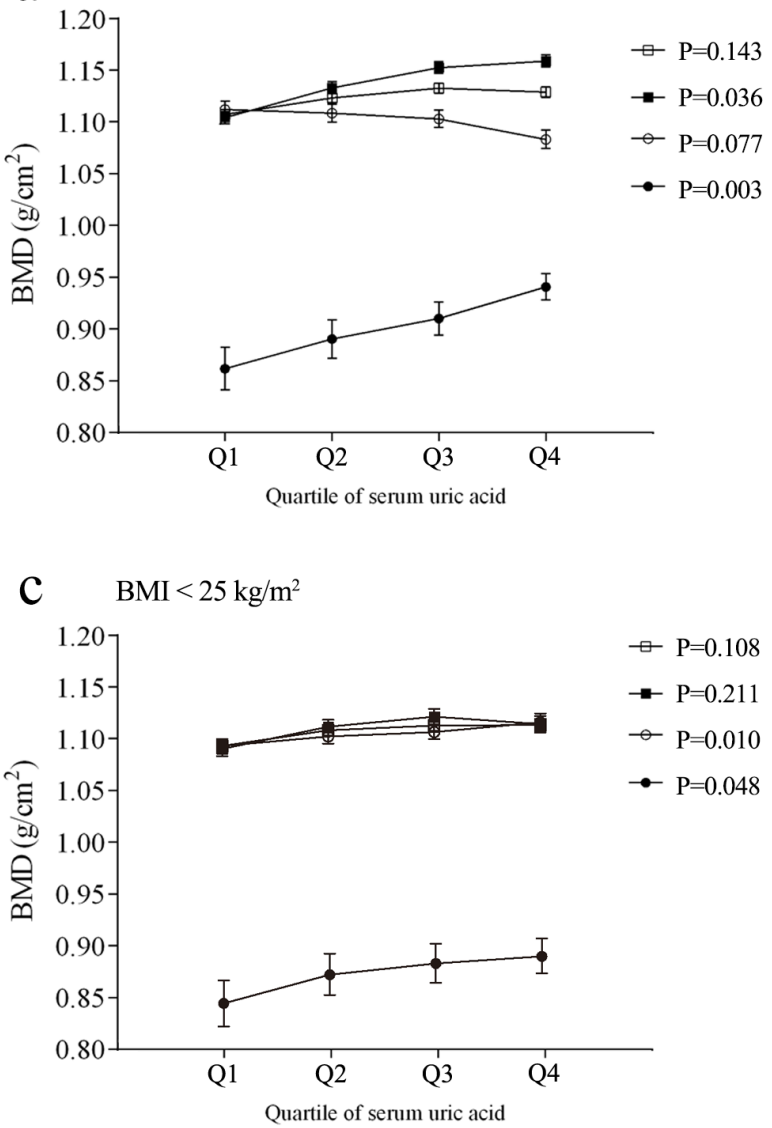

$\mathrm{b}$
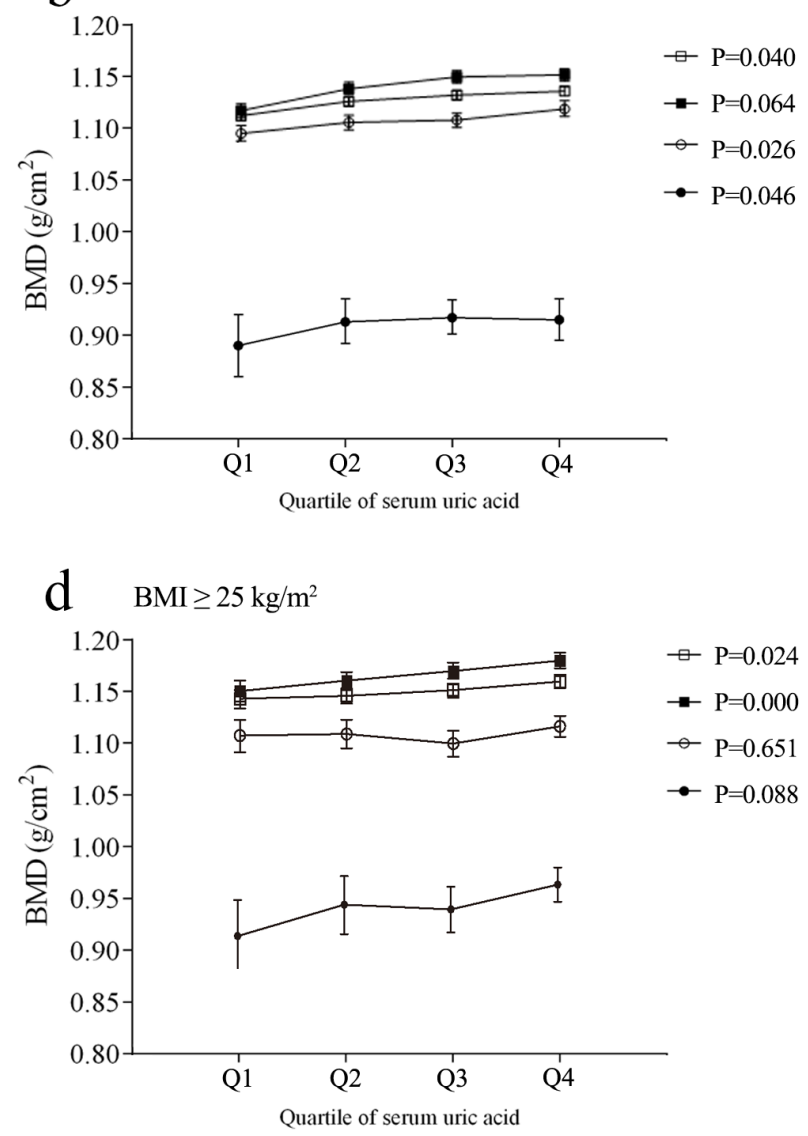

Figure 2: Bone mineral density (BMD) in each quartile of serum uric acid (Q1, Q2, Q3, and Q4). a. Unadjusted BMD. b. BMD adjusted for age; height; weight; systolic blood pressure; levels of total protein, serum calcium, serum phosphorus, alkaline phosphatase, blood glucose, and blood lipids; and liver function. c. Adjusted BMD in the body mass index (BMI) $<25$ group. d. Adjusted BMD in the BMI $\geq 25$ group. Results are presented as the survey weighted least-square means and $95 \%$ confidence intervals from regression analysis. $P$-values are from the test for a linear trend across the serum uric acid quartiles. 


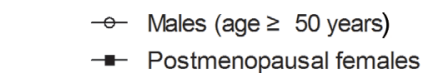

a

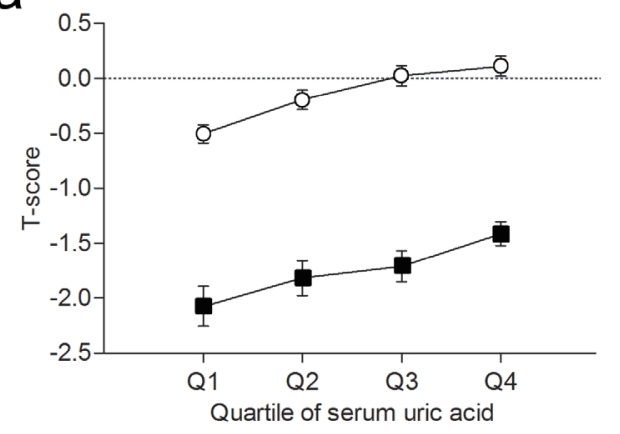

$\neg-$ Males (age $<50$ years)

- Premenopausal females

C

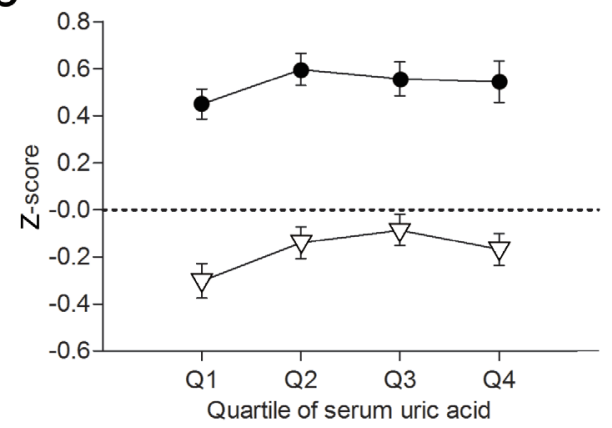

$-\mathrm{O} P=0.028$

$P=0.014$

$\neg P=0.359$

$P=0.498$ b

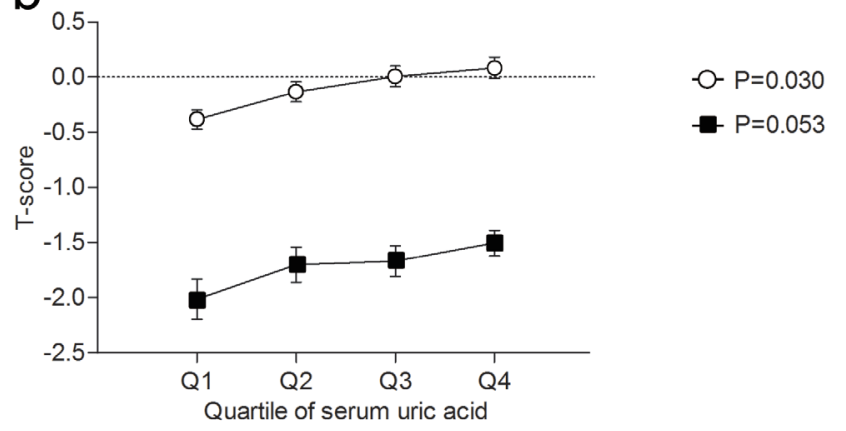

d

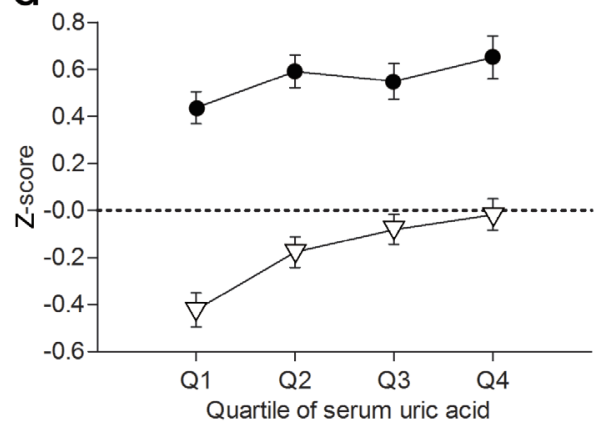

$\nabla P=0.049$

$P=0.134$

Figure 3: T-score and Z-score in each quartile of serum uric acid (Q1, Q2, Q3, and Q4) in male and female participants. a. Unadjusted T-score. b. T-score adjusted for age; height; weight; systolic blood pressure; levels of total protein, serum calcium, serum phosphorus, alkaline phosphatase, blood glucose, and blood lipids; and liver function. c. Unadjusted Z-score. d. Z-score adjusted for the abovementioned factors. Results are presented as the survey weighted least-square means and $95 \%$ confidence intervals from regression analysis. P-values are from the test for a linear trend across serum uric acid quartiles.

a. At least osteopenia $\quad$ OR $(95 \% \mathrm{CI})$

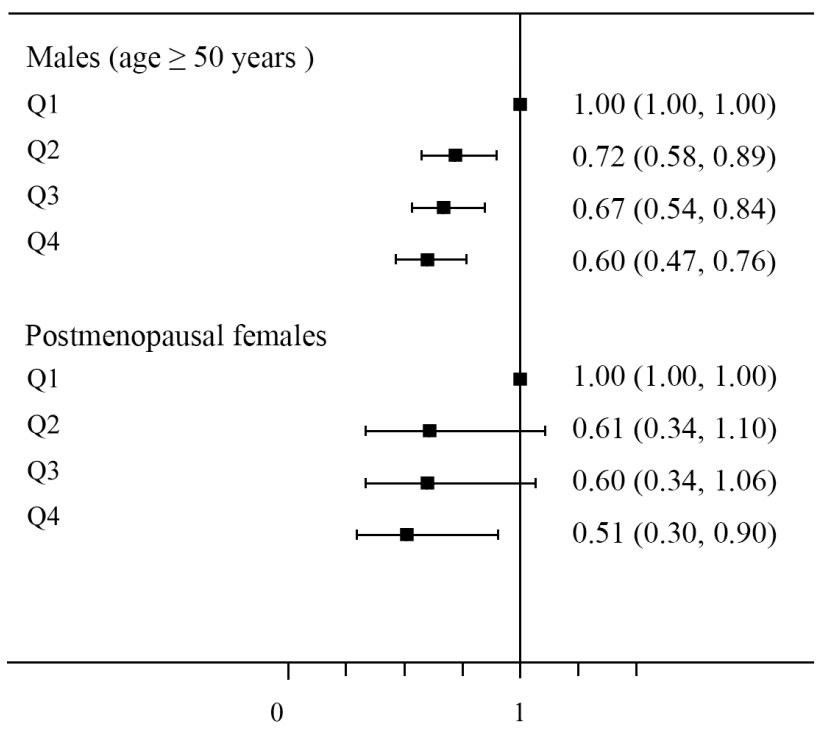

b. Osteoporosis

OR $(95 \% \mathrm{CI})$

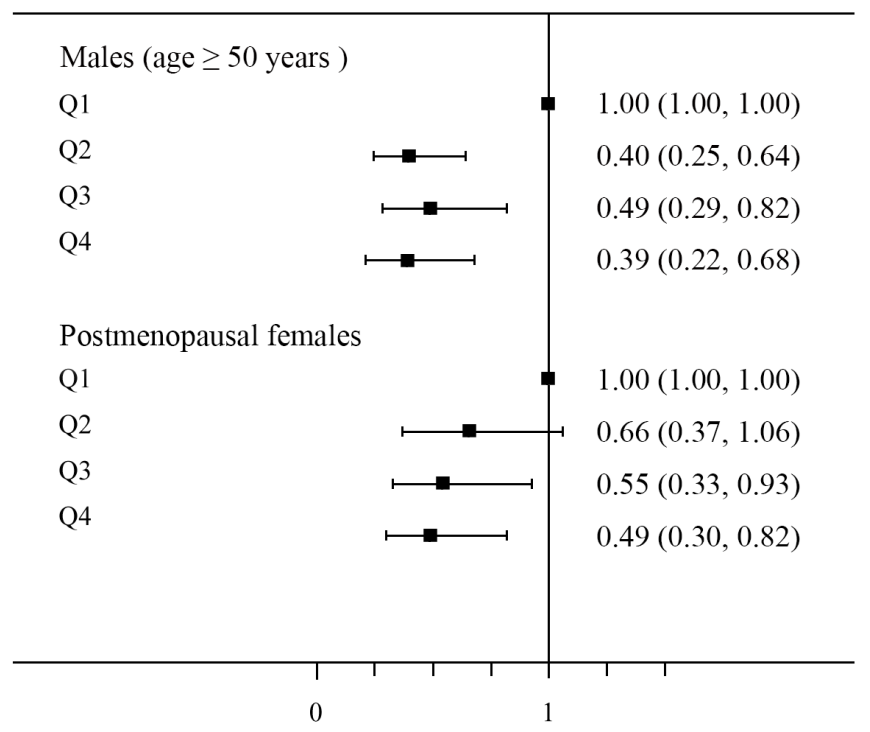

Figure 4: Odds ratios (ORs) and 95\% confidence intervals (CI) for a. at least osteopenia and b. osteoporosis according to the serum uric acid quartiles after adjusting for confounders. 
females for at least osteopenia and osteoporosis (Supplemental Figure 1).

\section{DISCUSSION}

The present study found that a high serum UA level was associated with a high BMD, T-score, and Z-score, and low ORs for at least osteopenia and osteoporosis.

Previous studies speculated that serum UA could serve as an antioxidant in patients with osteoporosis and could prevent the reduction of BMD in certain populations [17-21]. Recently, a study by Zhang D et al. did not show the protective effects of UA in a general US population, and the negative results were confirmed in an animal study [16]. The majority of these previous studies mainly focused on the association between serum UA levels and $\mathrm{BMD}$, and ignored other related variables, such as the T-score, Z-score, and prevalence of osteoporosis or osteopenia. The T-score and Z-score are considered key variables in the evaluation of bone loss.

In the present study, we found a positive association between serum UA levels and BMD after adjusting for confounders, which differs from the finding of the study performed by Zhang D et al. [16]. Additionally, further analysis demonstrated a positive interrelated relationship of serum UA levels with the T-score and Z-score. Moreover, the ORs for at least osteopenia and osteoporosis decreased from the low serum UA group to the high serum UA group in a dose-dependent manner. Furthermore, the protective effect of serum UA on bone health was confirmed in normal weight and overweight participants. These findings indicated that the trends were highly consistent independent of BMD, the T-score, the Z-score, at least osteopenia, and osteoporosis.

We comprehensively analyzed BMD, the T-score, the Z-score, and ORs for at least osteopenia and osteoporosis in this study. As with BMD, the T-score and Z-score might accurately and directly reflect the bone loss of specific individuals by considering age, sex, and ethnicity. Additionally, T-score-based subgroup (ORs for at least osteopenia and osteoporosis) analyses might help to amplify the minor differences in bone loss among different serum UA levels. Our study participants were recruited from five medical centers in China, and this might attenuate the population and regional variances in the present study.

Serum UA is a commonly measured biochemical parameter in health examinations and our results provide epidemiological evidence that serum UA might have a beneficial effect on bone health. Therefore, serum UA might be used to select individuals for DXA in order to assess osteoporosis or osteopenia.

The present study has several strengths. First, we collected data from 17,735 participants at five medical centers in China. The data included a broad spectrum of anthropometrics, laboratory assays and measurements, and
BMD measurement variables. Additionally, the present study included participants with a wide age range (20-90 years). Second, we performed a comprehensive analysis of the correlation between serum UA levels and bone loss. Furthermore, a dose-dependent protective effect of serum UA on bone loss was noted in our study.

The present study has some limitations. First, this was a cross-sectional study; therefore, it is difficult to conclude that there is a causal relationship between serum UA levels and bone loss. The follow-up of populations is important and meaningful. Second, fractures are one of the most common and serious complications of bone loss, and their incidence should be recorded in a further study. Third, this study did not evaluate dietary intake, such as intake of vegetables and fruits, which might have influenced bone health.

This large and multicenter general population-based study found that serum UA is associated with BMD, the $\mathrm{T}$-score, and the $\mathrm{Z}$-score, and has a strong protective effect against at least osteopenia and osteoporosis. Further studies are needed to elucidate the exact mechanisms by which serum UA contributes to bone loss.

\section{MATERIALS AND METHODS}

\section{Study population}

We performed a retrospective and consecutive cohort study from October 2009 to December 2014 at the following five medical centers: Sir Run Run Shaw Hospital Affiliated to Zhejiang University, Shanghai Ninth People's Hospital Affiliated to Shanghai Jiaotong University School of Medicine, First Affiliated Hospital of Wenzhou Medical University, Taizhou Enze Medical Center, and Shaoxing People's Hospital. The study participants consisted entirely of community individuals who visited these medical centers for health examinations and underwent extensive screening tests for the early detection of diseases, such as malignancy and osteoporosis. A total of 22,409 participants were enrolled. Participants diagnosed with diabetes mellitus, hypertension, hyperthyroidism, rheumatoid arthritis, gout, or urinary stones were excluded. Additionally, participants with a history of alcohol consumption (more than $20 \mathrm{~g}$ alcohol per day with current or past) or smoking (more than 10 cigarettes per day with current or past) and women with unknown menopausal status (MS) were excluded. Moreover, participants with abnormal results in liver, renal, or thyroid function tests were excluded from this study. Furthermore, participants on medications that could influence bone metabolism, such as diuretics, estrogens, and bisphosphonates, for a long period ( $>3$ months) were excluded. This study was approved by the ethics committees of all five medical centers, and the 
requirement of informed consent was waived owing to the retrospective nature of the study.

\section{Participant baseline characteristics}

Data on the baseline characteristics of the participant were collected. Age, smoking history, alcohol consumption history, medication history, MS, and medical history were recorded using a standardized questionnaire. MS was categorized as follows: 1, premenopausal (regular menstrual cycles); 2, perimenopausal (menses or amenorrhea of at least three months but less than 12 months); and 3, postmenopausal (amenorrhea for 12 consecutive months). BP was measured in the resting state with a standard mercury sphygmomanometer. Height and weight were measured by trained nurses, with participants wearing a light gown, and BMI was calculated for all participants.

\section{BMD measurements}

Areal BMD $\left(\mathrm{g} / \mathrm{cm}^{2}\right)$ of the lumbar spine (L1 to L4) was measured using DXA with Lunar equipment (Prodigy; Lunar, Madison, WI). All measurements were taken by experienced operators on the same parameter settings following standardized procedures. A standard quality control (QC) program, including daily calibrations with machine-specific phantoms, was used in the medical centers. A total of five Lunar devices were used in this study, and they were cross-calibrated. The same densitometer was used throughout the study. The performance of the DXA scanner was monitored throughout the study. Routine daily QC scans of a spine phantom were performed, and the coefficients of variation $(\mathrm{CVs})$ for QC BMD measurements in the five medical centers were $0.91 \%, 0.85 \%, 0.82 \%, 0.95 \%$, and $0.89 \%$. In vivo reproducibilities were estimated from duplicate scans (60 patients with repositioning between scans in each medical center) as the CVs for BMD. The CVs for lumbar spine BMD in the five medical centers were $1.15 \%, 1.07 \%, 0.95 \%, 1.22 \%$, and $1.01 \%$. The BMD measurements provided absolute values for the lumbar spine, and the BMD values were compared with those of healthy young Chinese adults of the same sex and ethnicity (T-score) or an age-, sex-, and ethnicitymatched reference population (Z-score). According to the World Health Organization, the T-score is the relevant measure when screening for osteoporosis and osteopenia in postmenopausal women and men aged $\geq 50$ years. The classification criteria of the World Health Organization are as follows: normal (T-score $\geq-1.0)$, osteopenia $(-1.0>$ T-score $>-2.5)$, and osteoporosis (T-score $\leq-2.5)$. In this study, participants with osteoporosis and osteopenia were classified as "at least osteopenia."

\section{Laboratory assays and measurements}

During the health examinations, routine blood biochemical tests, including serum UA, total protein, blood urea nitrogen, serum calcium, serum phosphorus, alkaline phosphatase, liver function, blood glucose, and blood lipids, were performed. The liver function tests included ALT, AST, and total bilirubin. The blood lipid tests included total cholesterol, low-density lipoprotein cholesterol, high-density lipoprotein cholesterol, and triglycerides. The eGFR was calculated according to age, sex, race/ethnicity, and the serum creatinine level using the Chronic Kidney Disease Epidemiology Collaboration (CKD-EPI) equation, which has been shown to perform well in the Chinese population $[23,24]$. The CKD-EPI equation is as follows: GFR $\left(\mathrm{mL} / \mathrm{min} / 1.73 \mathrm{~m}^{2}\right)=141 \times$ $\min (\mathrm{Scr} / \kappa, 1)^{\alpha} \times \max (\mathrm{Scr} / \kappa, 1)^{-1.209} \times 0.993^{\mathrm{Age}} \times 1.018$ [if female] $\times 1.159$ [if black] ( $\kappa$ : male, 0.9 ; female, 0.7 and $\alpha$ : male, -0.411 ; female, -0.329$)$. The participants were divided into quartiles according to the serum UA levels as follows: Q1: $\leq 320 \mathrm{mmol} / \mathrm{L}, \mathrm{Q} 2: 321-370 \mathrm{mmol} / \mathrm{L}, \mathrm{Q} 3$ : $371-425 \mathrm{mmol} / \mathrm{L}$, and Q4 $\geq 426 \mathrm{mmol} / \mathrm{L}$ for males, and $\mathrm{Q} 1 \leq 220 \mathrm{mmol} / \mathrm{L}, \mathrm{Q} 2: 221-260 \mathrm{mmol} / \mathrm{L}$, Q3: 261-300 $\mathrm{mmol} / \mathrm{L}$, and Q4 $\geq 301 \mathrm{mmol} / \mathrm{L}$ for females.

\section{Statistical analysis}

The data are presented as means \pm standard deviations or percentages. Categorical variables were compared using the $\chi^{2}$ test or Fisher's exact test, as appropriate, and continuous variables were compared using the Wilcoxon signed rank test. The relationships of serum UA levels with BMD, the T-score, the Z-score, osteopenia, and osteoporosis were investigated after controlling for various confounders. The potential confounding factors included age; height; weight; systolic BP; levels of total protein, serum calcium, serum phosphorus, alkaline phosphatase, blood glucose, and blood lipids; and liver function. Linear regression analyses and covariance analyses were performed to estimate the trends of the mean BMD, T-score, and Z-score across the increasing subgroup-specific quartiles of serum UA in unadjusted and multivariable-adjusted models. Binary logistic regression analyses were performed to determine the correlation of serum UA levels with osteoporosis and at least osteopenia after adjusting for potential confounders. ORs and corresponding 95\% CI were calculated. All statistical analyses were performed using SPSS 16.0 software (IBM, Armonk, NY). A two-sided $P$-value $<0.05$ was considered statistically significant.

\section{CONFLICTS OF INTEREST}

Xianfeng Lin, Chenchen Zhao, An Qin, Dun Hong, Wenyue Liu, Kangmao Huang, Jian Mo, Hejun Yu, 
Shengjie Wu, and Shunwu Fan declare that they have no conflict of interest

\section{GRANT SUPPORT}

The project was supported by grant from the National Natural Science Foundation of China (Grant No. 81201364), grant sponsored by shanghai pujiang program (14PJ1406200), innovative program from shanghai municipal education commission (13YZ031) and grant from 985 translational medicine program of Shanghai Jiaotong University, School of medicine (TS201317 AND TS201418).

\section{Disclosures}

No benefits in any form have been or will be received from a commercial party related directly or indirectly to the subject of this manuscript.

\section{REFERENCES}

1. Johnson RJ, Kang DH, Feig D, Kivlighn S, Kanellis J, Watanabe S, Tuttle KR, Rodriguez-Iturbe B, HerreraAcosta $\mathrm{J}$ and Mazzali M. Is there a pathogenetic role for uric acid in hypertension and cardiovascular and renal disease? Hypertension. 2003; 41:1183-1190.

2. Dehghan A, van Hoek M, Sijbrands EJ, Hofman A and Witteman JC. High serum uric acid as a novel risk factor for type 2 diabetes. Diabetes Care. 2008; 31:361-362.

3. Holme I, Aastveit AH, Hammar N, Jungner I and Walldius G. Uric acid and risk of myocardial infarction, stroke and congestive heart failure in 417,734 men and women in the Apolipoprotein MOrtality RISk study (AMORIS). J Intern Med. 2009; 266:558-570.

4. Verdecchia P, Schillaci G, Reboldi G, Santeusanio F, Porcellati $\mathrm{C}$ and Brunetti P. Relation between serum uric acid and risk of cardiovascular disease in essential hypertension. The PIUMA study. Hypertension. 2000; 36:1072-1078.

5. Bergamini C, Cicoira M, Rossi A and Vassanelli C. Oxidative stress and hyperuricaemia: pathophysiology, clinical relevance, and therapeutic implications in chronic heart failure. Eur J Heart Fail. 2009; 11:444-452.

6. Massa J, O'Reilly E, Munger KL, Delorenze GN and Ascherio A. Serum uric acid and risk of multiple sclerosis. J Neurol. 2009; 256:1643-1648.

7. Keizman D, Ish-Shalom M, Berliner S, Maimon N, Vered Y, Artamonov I, Tsehori J, Nefussy B and Drory VE. Low uric acid levels in serum of patients with ALS: further evidence for oxidative stress? J Neurol Sci. 2009; 285:9599.

8. Andreadou E, Nikolaou C, Gournaras F, Rentzos M, Boufidou F, Tsoutsou A, Zournas C, Zissimopoulos V and
Vassilopoulos D. Serum uric acid levels in patients with Parkinson's disease: their relationship to treatment and disease duration. Clin Neurol Neurosurg. 2009; 111:724728.

9. Euser SM, Hofman A, Westendorp RG and Breteler MM. Serum uric acid and cognitive function and dementia. Brain. 2009; 132:377-382.

10. Manolagas SC. From estrogen-centric to aging and oxidative stress: a revised perspective of the pathogenesis of osteoporosis. Endocr Rev. 2010; 31:266-300.

11. Varacallo MA and Fox EJ. Osteoporosis and its complications. Med Clin North Am. 2014; 98:817-831, xiixiii.

12. Maggio D, Barabani M, Pierandrei M, Polidori MC, Catani M, Mecocci P, Senin U, Pacifici R and Cherubini A. Marked decrease in plasma antioxidants in aged osteoporotic women: results of a cross-sectional study. J Clin Endocrinol Metab. 2003; 88:1523-1527.

13. Sugiura M, Nakamura M, Ogawa K, Ikoma Y, Ando F and Yano M. Bone mineral density in post-menopausal female subjects is associated with serum antioxidant carotenoids. Osteoporos Int. 2008; 19:211-219.

14. Sugiura M, Nakamura M, Ogawa K, Ikoma Y, Ando F, Shimokata $\mathrm{H}$ and Yano M. Dietary patterns of antioxidant vitamin and carotenoid intake associated with bone mineral density: findings from post-menopausal Japanese female subjects. Osteoporos Int. 2011; 22:143-152.

15. Sendur OF, Turan Y, Tastaban E and Serter M. Antioxidant status in patients with osteoporosis: a controlled study. Joint Bone Spine. 2009; 76:514-518.

16. Zhang D, Bobulescu IA, Maalouf NM, Adams-Huet B, Poindexter J, Park S, Wei F, Chen C, Moe OW and Sakhaee K. Relationship Between Serum Uric Acid and Bone Mineral Density in the General Population and in Rats with Experimental Hyperuricemia. J Bone Miner Res. 2015; 30:992-9.

17. Nabipour I, Sambrook PN, Blyth FM, Janu MR, Waite LM, Naganathan V, Handelsman DJ, Le Couteur DG, Cumming RG and Seibel MJ. Serum uric acid is associated with bone health in older men: a cross-sectional population-based study. J Bone Miner Res. 2011; 26:955-964.

18. Ahn SH, Lee SH, Kim BJ, Lim KH, Bae SJ, Kim EH, Kim HK, Choe JW, Koh JM and Kim GS. Higher serum uric acid is associated with higher bone mass, lower bone turnover, and lower prevalence of vertebral fracture in healthy postmenopausal women. Osteoporos Int. 2013; 24:2961-2970.

19. Makovey J, Macara M, Chen JS, Hayward CS, March L, Seibel MJ and Sambrook PN. Serum uric acid plays a protective role for bone loss in peri- and postmenopausal women: a longitudinal study. Bone. 2013; 52:400-406.

20. Ishii S, Miyao M, Mizuno $\mathrm{Y}$, Tanaka-Ishikawa M, Akishita $\mathrm{M}$ and Ouchi Y. Association between serum uric acid and lumbar spine bone mineral density in peri- and 
postmenopausal Japanese women. Osteoporos Int. 2014; 25:1099-1105.

21. Kim BJ, Baek S, Ahn SH, Kim SH, Jo MW, Bae SJ, Kim HK, Choe J, Park GM, Kim YH, Lee SH, Kim GS and Koh JM. Higher serum uric acid as a protective factor against incident osteoporotic fractures in Korean men: a longitudinal study using the National Claim Registry. Osteoporos Int. 2014; 25:1837-1844.

22. Lane NE, Parimi N, Lui LY, Wise BL, Yao W, Lay YA, Cawthon PM and Orwoll E. Association of serum uric acid and incident nonspine fractures in elderly men: the Osteoporotic Fractures in Men (MrOS) study. J Bone Miner Res. 2014; 29:1701-1707.

23. Levey AS, Stevens LA, Schmid CH, Zhang YL, Castro AF, 3rd, Feldman HI, Kusek JW, Eggers P, Van Lente F, Greene $\mathrm{T}$ and Coresh J. A new equation to estimate glomerular filtration rate. Ann Intern Med. 2009; 150:604-612.

24. Kong X, Ma Y, Chen J, Luo Q, Yu X, Li Y, Xu J, Huang S, Wang L, Huang W, Wang M, Xu G, Zhang L, Zuo L and Wang H. Evaluation of the Chronic Kidney Disease Epidemiology Collaboration equation for estimating glomerular filtration rate in the Chinese population. Nephrol Dial Transplant. 2013; 28:641-651. 